\title{
Normal Families of Möbius Maps
}

\author{
A. F. Beardon ${ }^{1}$. D. Minda ${ }^{2} \cdot$ I. Short ${ }^{3}$
}

Received: 13 January 2020 / Revised: 16 April 2020 / Accepted: 7 May 2020 / Published online: 17 July 2020 (c) The Author(s) 2020

\begin{abstract}
Several necessary and sufficient conditions for a family of Möbius maps to be a normal family in the extended complex plane $\mathbb{C}_{\infty}$ are established. Each of these conditions involves collections of two or three points which may vary with the Möbius maps in the family, provided the points satisfy a uniform separation condition. In addition, we derive a sufficient condition for the normality of a family of Möbius maps in terms of the average value of the reciprocal of the chordal derivative.
\end{abstract}

Keywords Chordal derivative $\cdot$ Meromorphic $\cdot$ Möbius maps $\cdot$ Normal families

Mathematics Subject Classification 30D45 $\cdot 51 \mathrm{~B} 10 \cdot 51 \mathrm{M} 10$

\section{Introduction}

We establish several necessary and sufficient conditions for a family of Möbius maps to be a normal family in the extended complex plane $\mathbb{C}_{\infty}$. Each of these conditions involves collections of two or three points that satisfy a uniform separation condition. These conditions are refinements of known characterisations of normal families of

Dedicated to the memory of our colleague Stephan Ruscheweyh.

Communicated by Filippo Bracci.

$\bowtie$ I. Short

ian.short@open.ac.uk

A. F. Beardon

afb@dpmms.cam.ac.uk

D. Minda

minda@ucmail.uc.edu

1 Centre for Mathematical Sciences, Wilberforce Road, Cambridge CB3 0WB, UK

2 Department of Mathematical Sciences, University of Cincinnati, Ohio 45221-0025, USA

3 School of Mathematics and Statistics, The Open University, Milton Keynes MK7 6AA, UK 
meromorphic functions. Despite the perceived elementary nature of Möbius maps, and the familiarity of normal families in complex analysis, we believe that the role of Möbius maps in normal families is not properly represented in the literature, so it may be useful to record the following discussion.

A family $\mathcal{F}$ of functions, each meromorphic in a region $\Omega$ in $\mathbb{C}_{\infty}$, is a normal family, or (more briefly) is normal, in $\Omega$ if each sequence taken from $\mathcal{F}$ has a subsequence that converges, uniformly with respect to the chordal metric $\chi$, on each compact subset of $\Omega$ to some meromorphic function (which may be the constant function $\infty$ ); see, for example, $[6,9,14,15,20]$. The importance of normal families in complex analysis is beyond doubt, and this stems largely from Montel's famous three omitted values theorem: a family $\mathcal{F}$ of functions, each meromorphic in a region $\Omega$, is normal in $\Omega$ if there are three distinct values $u, v$ and $w$ such that, for each $f$ in $\mathcal{F}$, none of the equations $f(z)=u, f(z)=v$ and $f(z)=w$ has a solution $z$ in $\Omega$. In [8, p. 202] Carathéodory extended Montel's result to allow the omitted values to depend on the function providing that these values satisfy a suitable separation condition which is given in terms of a product of three chordal distances. See [6, Thm. 8.4] for a recent proof of Carathéodory's theorem.

Carathéodory's Theorem $A$ family $\mathcal{F}$ of functions, each meromorphic in a region $\Omega$, is normal in $\Omega$ if, for each $f$ in $\mathcal{F}$, there are three distinct values $u_{f}, v_{f}$ and $w_{f}$ such that $f$ omits these values in $\Omega$, and

$$
\inf \left\{\chi\left(u_{f}, v_{f}\right) \chi\left(v_{f}, w_{f}\right) \chi\left(w_{f}, u_{f}\right): f \in \mathcal{F}\right\}>0
$$

Here $\chi$ denotes the chordal distance on $\mathbb{C}_{\infty}$. The use of the product here is a distraction, for as $\chi \leq 2$, inequality (1.1) is equivalent to the three inequalities

$$
\begin{aligned}
& \inf \left\{\chi\left(u_{f}, v_{f}\right): f \in \mathcal{F}\right\}>0 ; \\
& \inf \left\{\chi\left(v_{f}, w_{f}\right): f \in \mathcal{F}\right\}>0 ; \\
& \inf \left\{\chi\left(w_{f}, u_{f}\right): f \in \mathcal{F}\right\}>0 .
\end{aligned}
$$

A family of triples $\left\{\left(u_{f}, v_{f}, w_{f}\right): f \in \mathcal{F}\right\}$ satisfying the three inequalities above is said to be uniformly separated, and the same phrase is used to describe a family of pairs $\left\{\left(u_{f}, v_{f}\right): f \in \mathcal{F}\right\}$ satisfying just the first inequality above.

Despite the importance of Montel's three omitted values theorem, the existence of three omitted values is not a necessary condition for normality (consider the functions $z \mapsto z+n, n=1,2, \ldots$, on $\mathbb{C}$ ), and much later Montel improved this result by giving a necessary and sufficient condition for the normality of a family of meromorphic maps [16]. This later result, which does not seem to be as well known as it might be, requires that there are four points in the codomain whose inverse image sets, which need not be non-empty, are uniformly separated on compact subsets of $\Omega$. This result contains the three omitted values theorem as a special case, although the proof in [16] contains an error which has been corrected in [7]. We note that whereas Carathéodory's result requires the separation of points in the codomain, Montel's result requires the separation of points in the domain $\Omega$. This apparent discrepancy will be resolved later. For Möbius maps four points can be reduced to three points. 
Theorem 1 For any family $\mathcal{F}$ of Möbius maps the following are equivalent.

(a) The family $\mathcal{F}$ is normal in $\mathbb{C}_{\infty}$.

(b) For any uniformly separated set of triples $\left\{\left(u_{f}, v_{f}, w_{f}\right): f \in \mathcal{F}\right\}$, the set $\left\{\left(f^{-1}\left(u_{f}\right), f^{-1}\left(v_{f}\right), f^{-1}\left(w_{f}\right)\right): f \in \mathcal{F}\right\}$ is also uniformly separated.

(c) There exists a uniformly separated set of triples $\left\{\left(u_{f}, v_{f}, w_{f}\right): f \in \mathcal{F}\right\}$ such that $\left\{\left(f^{-1}\left(u_{f}\right), f^{-1}\left(v_{f}\right), f^{-1}\left(w_{f}\right)\right): f \in \mathcal{F}\right\}$ is uniformly separated.

Moreover, [7] contains a local three omitted values characterization of normality as follows. Suppose that $A$ is a set of four points in $\mathbb{C}_{\infty}$. A family $\mathcal{F}$ of meromorphic functions is normal in a region $\Omega$ if and only if each point $z_{0} \in \Omega$ has a neighbourhood $U_{0}$ in which each $f$ in $\mathcal{F}$ omits at least three of the four values in $A$. For Möbius maps the cardinality of the set $A$ can be decreased from four to three. Loosely speaking, the next result says that a family $\mathcal{F}$ of Möbius maps is normal if and only if each $f$ in $\mathcal{F}$ locally omits two of three given values.

Theorem 2 For any family $\mathcal{F}$ of Möbius maps and any region $\Omega$ in $\mathbb{C}_{\infty}$ the following are equivalent.

(a) The family $\mathcal{F}$ is normal in $\Omega$.

(b) For any set $A=\{u, v, w\}$ of distinct points in $\mathbb{C}_{\infty}$, each point $z_{0}$ in $\Omega$ has a neighbourhood in which each $f$ in $\mathcal{F}$ omits at least two of the values in $A$.

(c) There exists a set $A=\{u, v, w\}$ of distinct points in $\mathbb{C}_{\infty}$ such that each point $z_{0}$ in $\Omega$ has a neighbourhood in which each $f$ in $\mathcal{F}$ omits at least two of the values in $A$.

Next, Hinkkanen [10] and Lappan [12] independently showed that a family $\mathcal{F}$ of functions, meromorphic in a region $\Omega$, is normal in $\Omega$ if there are five distinct values $w_{1}, \ldots, w_{5}$ such that the family $\left\{f^{\#}: f \in \mathcal{F}\right\}$ of spherical derivatives of functions in $\mathcal{F}$ is bounded on the union of the five inverse-image sets $\left\{f^{-1}\left(w_{j}\right): f \in \mathcal{F}\right\}$. Previously, Lappan [11] had given a similar five-value characterization of normal functions on the unit disc. Here we show that for families of Möbius maps, and with the chordal derivative $f^{\chi}$ rather than the spherical derivative $f^{\#}$ (the choice of derivatives will be discussed later), the number five is reduced to two.

Theorem 3 For any family $\mathcal{F}$ of Möbius maps the following are equivalent.

(a) The family $\mathcal{F}$ is normal in $\mathbb{C}_{\infty}$.

(b) For any uniformly separated set of pairs $\left\{\left(u_{f}, v_{f}\right): f \in \mathcal{F}\right\}$,

$$
\sup \left\{f^{\chi}(z): f \in \mathcal{F}, z \in f^{-1}\left(\left\{u_{f}, v_{f}\right\}\right)\right\}<+\infty \text {. }
$$

(c) There exists a uniformly separated set of pairs $\left\{\left(u_{f}, v_{f}\right): f \in \mathcal{F}\right\}$ with

$$
\sup \left\{f^{\chi}(z): f \in \mathcal{F}, z \in f^{-1}\left(\left\{u_{f}, v_{f}\right\}\right)\right\}<+\infty \text {. }
$$

Beyond this, in Sect. 8 we will also derive a sufficient condition for the normality of a family of Möbius maps in terms of the average value of the reciprocals of the chordal derivatives taken over $\mathbb{C}_{\infty}$; see Lemma 1 and Theorem 10 . This result raises the question of whether or not some such averaging result holds for families of rational, or even meromorphic, maps. 


\section{Preliminary Material on Möbius Maps}

Throughout, $\mathbb{C}$ is the complex plane, which we identify with the co-ordinate plane $\left\{\left(x_{1}, x_{2}, x_{3}\right): x_{3}=0\right\}$ in $\mathbb{R}^{3}$ in the usual way, and $\mathbb{C}_{\infty}$ is the extended complex plane. The unit sphere is denoted by $\mathbb{S}^{2}$ and the open unit ball by $\mathbb{B}^{3}$, each in $\mathbb{R}^{3}$. Now the stereographic projection of $\mathbb{S}^{2}$ onto $\mathbb{C}_{\infty}$ is well known, and has a long history dating back to Hipparchus of Nicae (190-125 BCE) who may have been the first person to introduce the terms 'longitude' and 'latitude'. Apparently, the term 'stereographic projection' was introduced by F. D’ Aiguillon (1566-1617). However, we shall mainly use its inverse, which we denote by $\Phi$, and which is given by

$$
\Phi(z)=\left(\frac{2 x}{|z|^{2}+1}, \frac{2 y}{|z|^{2}+1}, \frac{|z|^{2}-1}{|z|^{2}+1}\right), \quad \Phi(\infty)=(0,0,1),
$$

where $x$ and $y$ are the real and imaginary parts of $z$, respectively. Note that if $\mathcal{I}$ is the inversion in the sphere in $\mathbb{R}^{3}$ with centre $(0,0,1)$ and radius $\sqrt{2}$, then stereographic projection and its inverse are simply the restrictions of $\mathcal{I}$ to $\mathbb{S}^{2}$ and $\mathbb{C}_{\infty}$, respectively. Finally, the chordal metric $\chi$ on $\mathbb{C}_{\infty}$ is defined by $\chi(z, w)=|\Phi(z)-\Phi(w)|$. It can be computed using the formulas

$$
\chi(z, w)=\frac{2|z-w|}{\sqrt{1+|z|^{2}} \sqrt{1+|w|^{2}}} \text { and } \chi(z, \infty)=\frac{2}{\sqrt{1+|z|^{2}}}
$$

for $z, w \neq \infty$.

A Möbius map is a map of the form

$$
f(z)=\frac{a z+b}{c z+d}, \quad a d-b c=1,
$$

where the norm $\|f\|$ of $f$ is defined (unambiguously) by

$$
\|f\|^{2}=|a|^{2}+|b|^{2}+|c|^{2}+|d|^{2}=\left\|f^{-1}\right\|^{2} .
$$

Note that because $a d-b c=1$,

$$
\begin{aligned}
\|f\|^{2}-2 & \geq|a|^{2}+|b|^{2}+|c|^{2}+|d|^{2}-2(|a d|+|b c|) \\
& =(|a|-|d|)^{2}+(|b|-|c|)^{2}
\end{aligned}
$$

so $\|f\|^{2} \geq 2$.

If $f$ is meromorphic at a point $z$ in $\mathbb{C}_{\infty}$, then the chordal derivative $f^{\chi}(z)$ of $f$ at $z$ is, by definition,

$$
f^{\chi}(z)=\lim _{w \rightarrow z} \frac{\chi(f(w), f(z))}{\chi(w, z)}
$$


This was introduced by Marty in his fundamental paper on normal families under the name derivée sphérique [13]. In more recent times, the spherical derivative has been defined by

$$
f^{\#}(z)=\frac{\left|f^{\prime}(z)\right|}{1+|f(z)|^{2}}=\lim _{w \rightarrow z} \frac{\chi(f(w), f(z))}{2|w-z|},
$$

and this should not be confused with our use of chordal derivative. Like Marty, we prefer to use the chordal derivative (rather than the modern spherical derivative) because it is more natural to use the same metric in both the domain and codomain, it allows either $z$ or $f(z)$ to be $\infty$, and (unlike the modern spherical derivative) it satisfies a chain rule which we will need later, namely $(f g)^{\chi}(z)=f^{\chi}(g(z)) g^{\chi}(z)$, where $f g$ denotes composition. Also, the chordal derivative of the identity map is the constant 1 , whereas the spherical derivative of the identity map is not even constant.

The chordal derivative of the Möbius map $f(z)=(a z+b) /(c z+d)$, where $a d-b c=1$, is

$$
f^{\chi}(z)=\frac{\left(1+|z|^{2}\right)\left|f^{\prime}(z)\right|}{1+|f(z)|^{2}}=\frac{1+|z|^{2}}{|a z+b|^{2}+|c z+d|^{2}},
$$

so

$$
\frac{1}{f^{\chi}(z)}=\frac{|a z+b|^{2}+|c z+d|^{2}}{1+|z|^{2}} .
$$

Next, we convert an elementary Euclidean identity for Möbius maps into its chordal form. For any Möbius map $f$ and all $z, w \in \mathbb{C}$,

$$
|f(z)-f(w)|=|z-w| \sqrt{\left|f^{\prime}(z)\right|\left|f^{\prime}(w)\right|},
$$

provided neither $f(z)$ nor $f(w)$ is $\infty$. By substituting formulas for the chordal distance and chordal derivative into this Euclidean identity, we obtain

$$
\chi(f(z), f(w))=\chi(z, w) \sqrt{f^{\chi}(z) f^{\chi}(w)},
$$

and in fact this equation is true for all $z, w \in \mathbb{C}_{\infty}$.

\section{Uniformly Bi-Lipschitz Families}

Each Möbius map is a bi-Lipschitz map of the metric space $\left(\mathbb{C}_{\infty}, \chi\right)$ onto itself (see, for example, $[3,6])$ and, for $z \neq w$, we have

$$
\frac{1}{\|f\|^{2}} \leq \frac{\chi(f(z), f(w))}{\chi(z, w)} \leq\|f\|^{2}
$$


The best inequality of this form is

$$
\frac{\chi(z, w)}{E(f)} \leq \chi(f(z), f(w)) \leq E(f) \chi(z, w),
$$

where

$$
E(f)=\frac{1}{2}\left(\|f\|^{2}+\sqrt{\|f\|^{4}-4}\right)
$$

(see $[3,4]$ ) and the (geometric) reason for this will be given later.

Observe that we have an infinitesimal version of (3.1), namely

$$
\frac{1}{\|f\|^{2}} \leq f^{\chi}(z) \leq\|f\|^{2}
$$

and since $\|f\|=\left\|f^{-1}\right\|$, we also have

$$
\frac{1}{\|f\|^{2}} \leq \frac{\chi\left(f^{-1}(z), f^{-1}(w)\right)}{\chi(z, w)} \leq\|f\|^{2}, \quad \frac{1}{\|f\|^{2}} \leq\left(f^{-1}\right)^{\chi}(z) \leq\|f\|^{2} .
$$

In any event, since $\|f\|=\left\|f^{-1}\right\|$ and $\mathbb{C}_{\infty}$ is compact, these inequalities together with the Arzelà-Ascoli theorem (see [6, Thm. 5.3]) yield the following most basic result of all; here $\mathcal{F}^{-1}=\left\{f: f^{-1} \in \mathcal{F}\right\}$.

Theorem 4 A family $\mathcal{F}$ of Möbius maps is normal in $\mathbb{C}_{\infty}$ if and only if any one (and hence all) of the following conditions hold:

(a) $\mathcal{F}$ is a uniformly bi-Lipschitz family of self-maps of $\left(\mathbb{C}_{\infty}, \chi\right)$;

(b) $\sup \{\|f\|: f \in \mathcal{F}\}<+\infty$;

(c) $\mathcal{F}^{-1}$ is normal in $\mathbb{C}_{\infty}$.

Because $\left\|f^{-1}\right\|=\|f\|$ and $\|f g\| \leq\|f\|\|g\|$ for Möbius maps $f$ and $g$ (see [3, p. 12]), Theorem 4 has the following corollary.

Corollary 1 Suppose that $\mathcal{F}=\left\{f_{j}: j \in J\right\}$ and $\mathcal{G}=\left\{g_{j}: j \in J\right\}$ are normal families of Möbius maps in $\mathbb{C}_{\infty}$. Then $\left\{f_{j}^{-1}: j \in J\right\},\left\{f_{j} g_{j}: j \in J\right\}$ and $\left\{f_{j} g_{j}^{-1}: j \in J\right\}$ are also normal families in $\mathbb{C}_{\infty}$.

The first inequality in (3.1) leads to a proof of the following fact which is part of the folk-lore of the subject: if $\left(f_{n}\right)$ is a sequence of Möbius maps that converges uniformly with respect to $\chi$ on $\mathbb{C}_{\infty}$ to the function $f$, then $f$ is also a Möbius map. Indeed, as the $f_{n}$ are meromorphic throughout $\mathbb{C}_{\infty}$ we see that $f$ is meromorphic there; thus $f$ is a rational map. Next, $\left\{f_{1}, f_{2}, \ldots\right\}$ is a normal family so Theorem 4(a) implies that there is a positive number $L$ such that $\chi\left(f_{n}(z), f_{n}(w)\right) \geq L \chi(z, w)$ for all $z$ and $w$ in $\mathbb{C}_{\infty}$. If we fix distinct $z$ and $w$ and then let $n$ tend to $\infty$, we find that $f(z) \neq f(w)$; thus $f$ is an injective rational map and so is a Möbius map. It follows from this that the family of Möbius maps is closed in the family of all continuous self-maps of $\mathbb{C}_{\infty}$ with the topology of uniform convergence relative to $\chi$ on $\mathbb{C}_{\infty}$. 
If we recall Carathéodory's theorem, then we see from (a) and (c) in Theorem 4 that the separation of points in the domain, or the codomain, is equivalent to the separation of their images, or pre-images, respectively. In short, the distinction between these two issues is, for Möbius maps, illusory.

\section{Separation of Points and Normality}

Recall that a set $\left\{\left(u_{j}, v_{j}, w_{j}\right): j \in J\right\}$ of triples of distinct points in $\mathbb{C}_{\infty}$ is uniformly separated if

$$
\inf _{j \in J} \chi\left(u_{j}, v_{j}\right)>0, \quad \inf _{j \in J} \chi\left(v_{j}, w_{j}\right)>0, \quad \inf _{j \in J} \chi\left(w_{j}, u_{j}\right)>0 .
$$

If this is so then we let

$$
\chi\left[u_{j}, v_{j}, w_{j}: j \in J\right]=\inf \left\{\chi\left(u_{j}, v_{j}\right) \chi\left(v_{j}, w_{j}\right) \chi\left(w_{j}, u_{j}\right): j \in J\right\},
$$

which is positive.

Similarly, a collection $\left\{\left(u_{j}, v_{j}\right): j \in J\right\}$ of pairs of points is uniformly separated if $\inf _{j \in J} \chi\left(u_{j}, v_{j}\right)>0$.

The norm of a Möbius map can be expressed in terms of the images of the cube roots of unity.

Theorem 5 If $f$ is a Möbius map, then

$$
\|f\|^{2}=\frac{2}{\sqrt{3}} \frac{\chi(f(1), f(\omega))^{2}+\chi\left(f(\omega), f\left(\omega^{2}\right)\right)^{2}+\chi\left(f\left(\omega^{2}\right), f(1)\right)^{2}}{\chi(f(1), f(\omega)) \chi\left(f(\omega), f\left(\omega^{2}\right)\right) \chi\left(f\left(\omega^{2}\right), f(1)\right)},
$$

where $\omega=\exp (2 \pi i / 3)$.

Proof First, we derive an expression equivalent to the right-hand side of (4.1). Formula (2.3) implies that the numerator of the rightmost fraction in (4.1) is

$$
3\left(f^{\chi}(1) f^{\chi}(\omega)+f^{\chi}(\omega) f^{\chi}\left(\omega^{2}\right)+f^{\chi}\left(\omega^{2}\right) f^{\chi}(1)\right)
$$

and the denominator is

$$
3 \sqrt{3} f^{\chi}(1) f^{\chi}(\omega) f^{\chi}\left(\omega^{2}\right)
$$

because $\chi(1, \omega)=\chi\left(\omega, \omega^{2}\right)=\chi\left(\omega^{2}, 1\right)=\sqrt{3}$. Therefore, the right-hand side of (4.1) is

$$
\frac{2}{3}\left(\frac{1}{f \chi(1)}+\frac{1}{f \chi(\omega)}+\frac{1}{f \chi\left(\omega^{2}\right)}\right) .
$$

Next, we rewrite (4.2) in terms of $\|f\|^{2}$. For $|z|=1$, (2.2) gives

$$
\frac{1}{f^{\chi}(z)}=\frac{1}{2}\left(\|f\|^{2}+2 \operatorname{Re}((a \bar{b}+c \bar{d}) z)\right) \text {. }
$$


If we replace $z$ by $1, \omega$ and $\omega^{2}$ in (4.3), and then add the three resulting identities, we obtain

$$
\frac{2}{3}\left(\frac{1}{f^{\chi}(1)}+\frac{1}{f^{\chi}(\omega)}+\frac{1}{f^{\chi}\left(\omega^{2}\right)}\right)=\|f\|^{2},
$$

because the sum of the cube roots of unity is zero. Hence, (4.1) holds.

Corollary 2 Let $\mathcal{F}$ be a family of Möbius maps.

(a) If $\mathcal{F}$ is a normal family in $\mathbb{C}_{\infty}$, say $\|f\| \leq N$ for all $f \in \mathcal{F}$, then

$$
\chi\left[f(1), f(\omega), f\left(\omega^{2}\right): f \in \mathcal{F}\right] \geq \frac{3 \sqrt{3}}{N^{6}} .
$$

(b) If $\varepsilon>0$ and $\chi\left[f(1), f(\omega), f\left(\omega^{2}\right): f \in \mathcal{F}\right] \geq \varepsilon$, then $\mathcal{F}$ is a normal family in $\mathbb{C}_{\infty}$ with $\|f\|^{2} \leq 8 \sqrt{3} / \varepsilon$ for all $f \in \mathcal{F}$.

Less precisely, a family of Möbius maps is normal if and only if the images of 1, $\omega$ and $\omega^{2}$ are uniformly separated over the family.

Proof The assertion in part (a) follows immediately from (3.1) because $\chi(1, \omega)=$ $\chi\left(\omega, \omega^{2}\right)=\chi\left(\omega^{2}, 1\right)=\sqrt{3}$. The proof of part (b) is also brief since identity (4.1) gives

$$
\|f\|^{2} \leq \frac{8 \sqrt{3}}{\chi(f(1), f(\omega)) \chi\left(f(\omega), f\left(\omega^{2}\right)\right) \chi\left(f\left(\omega^{2}\right), f(1)\right)} \leq \frac{8 \sqrt{3}}{\varepsilon}
$$

A larger upper bound for $\|f\|^{2}$ was given in [6, Thm. 8.2] using a different method of proof.

Remark 1 The right-hand side of (4.1) relates to Weitzenböck's inequality in Euclidean geometry [21]; see [17] for a simple proof of a more general result. In order to establish the connection, view $f(1), f(\omega)$ and $f\left(\omega^{2}\right)$ as points on the unit sphere $\mathbb{S}^{2}$. These three points determine a plane in $\mathbb{R}^{3}$ and a Euclidean triangle in this plane; let $A, B$, $C$ be the side lengths of this Euclidean triangle. Weitzenböck's inequality asserts that

$$
A^{2}+B^{2}+C^{2} \geq 4 \sqrt{3} I
$$

where $I$ denotes the area of the triangle, and equality holds if and only if the triangle is equilateral. Recall that the area of the triangle is given by $I=A B C /(4 R)$, where $R$ is the circumradius of the triangle. Hence, (4.6) gives

$$
\frac{2\left(A^{2}+B^{2}+C^{2}\right)}{\sqrt{3} A B C} \geq \frac{2}{R},
$$

with equality if and only if the triangle is equilateral. Hence, $\|f\|^{2} \geq 2 / R$. The vertices of the triangle lie on the unit sphere, so $R \leq 1$ and we obtain the known inequality $\|f\|^{2} \geq 2$ with equality if and only if $f(1), f(\omega), f\left(\omega^{2}\right)$ are the vertices of an equilateral triangle inscribed in a great circle (in which case $f$ is a rotation of $\mathbb{S}^{2}$ ). 
We come now to a result at the heart of the arguments about separated values (whether they be omitted values or pre-images). Recall that Möbius maps act triplytransitively on $\mathbb{C}_{\infty}$. In order to obtain the normality of a family of Möbius maps that carry prescribed triples to another set of prescribed triples, uniform separation of the triples is required.

Theorem 6 Let $\left\{\left(u_{j}, v_{j}, w_{j}\right): j \in J\right\}$ and $\left\{\left(p_{j}, q_{j}, r_{j}\right): j \in J\right\}$ be uniformly separated sets of triples of distinct points in $\mathbb{C}_{\infty}$ and, for each $j$, let $h_{j}$ be the unique Möbius map such that $h_{j}\left(u_{j}\right)=p_{j}, h_{j}\left(v_{j}\right)=q_{j}$ and $h_{j}\left(w_{j}\right)=r_{j}$. Then $\left\{h_{j}: j \in J\right\}$ is a normal family of Möbius maps in $\mathbb{C}_{\infty}$. Explicitly, for all $j$,

$$
\left\|h_{j}\right\| \leq \frac{8 \sqrt{3}}{\chi\left[u_{j}, v_{j}, w_{j}: j \in J\right]^{1 / 2} \chi\left[p_{j}, q_{j}, r_{j}: j \in J\right]^{1 / 2}} .
$$

This result combined with Corollary 1 implies that we can move from one uniformly separated set of triples to another (typically, one of these sets might be the special triple $\left.\left\{1, \omega, \omega^{2}\right\}\right)$ by the use of a normal family $\left\{h_{j}\right\}$ of Möbius maps. For example, in such arguments we can replace three given omitted values by three uniformly separated values without creating or destroying normality.

Proof It is obvious from Corollary 2 that if $\left\{\left(u_{j}, v_{j}, w_{j}\right): j \in J\right\}$ is a uniformly separated set of triples of distinct points in $\mathbb{C}_{\infty}$ and if, for each $j, f_{j}$ is the unique Möbius map such that $f_{j}\left(u_{j}\right)=1, f_{j}\left(v_{j}\right)=\omega$ and $f_{j}\left(w_{j}\right)=\omega^{2}$, then $\left\{f_{j}: j \in J\right\}$ is a normal family of Möbius maps in $\mathbb{C}_{\infty}$; indeed, from Corollary 2(b),

$$
\left\|f_{j}\right\|^{2} \leq \frac{8 \sqrt{3}}{\chi\left[u_{j}, v_{j}, w_{j}: j \in J\right]}
$$

Similarly, if $g_{j}$ is the Möbius map such that $g_{j}\left(p_{j}\right)=1, g_{j}\left(q_{j}\right)=\omega$ and $g_{j}\left(r_{j}\right)=\omega^{2}$, then

$$
\left\|g_{j}\right\|^{2} \leq \frac{8 \sqrt{3}}{\chi\left[p_{j}, q_{j}, r_{j}: j \in J\right]} .
$$

As $h_{j}=g_{j}^{-1} f_{j}$, we have $\left\|h_{j}\right\|=\left\|g_{j}^{-1} f_{j}\right\| \leq\left\|g_{j}^{-1}\right\|\left\|f_{j}\right\|=\left\|g_{j}\right\|\left\|f_{j}\right\|$, and (4.8) follows from this.

Essentially the same proof shows that the following result is also true (alternatively, we can convert the uniformly separated pairs into uniformly separated triples and apply Theorem 6).

Corollary 3 Let $\left\{\left(u_{j}, v_{j}\right): j \in J\right\}$ and $\left\{\left(p_{j}, q_{j}\right): j \in J\right\}$ be uniformly separated sets of pairs of distinct points in $\mathbb{C}_{\infty}$. Then there exists a normal family $\left\{h_{j}: j \in J\right\}$ of Möbius maps in $\mathbb{C}_{\infty}$ such that, for each $j, h_{j}\left(u_{j}\right)=p_{j}$ and $h_{j}\left(v_{j}\right)=q_{j}$.

We can now prove Theorem 1 . 
Proof of Theorem 1 To show that (a) implies (b), suppose that the set of triples $\left(u_{f}, v_{f}, w_{f}\right)$ is uniformly separated and that $\mathcal{F}$ is normal. Then $\mathcal{F}^{-1}$ is normal, and so uniformly bi-Lipschitz. It follows that the set of triples $\left(f^{-1}\left(u_{f}\right), f^{-1}\left(v_{f}\right), f^{-1}\left(w_{f}\right)\right)$ is uniformly separated, so (a) implies (b).

That (b) implies (c) is trivial.

Finally, we show that (c) implies (a). Theorem 6 implies that if both sets $\left\{\left(u_{f}, v_{f}, w_{f}\right): f \in \mathcal{F}\right\}$ and $\left\{\left(f\left(u_{f}\right), f\left(v_{f}\right), f\left(w_{f}\right)\right): f \in \mathcal{F}\right\}$ are uniformly separated, then $\mathcal{F}$ is a normal family. The same holds if we replace $\mathcal{F}$ by $\mathcal{F}^{-1}$, so the hypotheses of (c) show that $\mathcal{F}^{-1}$ is normal. Thus $\mathcal{F}$ is also normal.

The next example demonstrates that the separated triples in Theorem 1 cannot be replaced with separated pairs.

Example 1 It is easy to see that the existence of two separated fibers does not imply normality. Let $\mathcal{F}=\left\{f_{1}, f_{2}, \ldots\right\}$ where $f_{n}=n z$, and, for each $f \in \mathcal{F}$, let $u_{f}=0$ and $v_{f}=\infty$. Then $\chi\left(u_{f}, v_{f}\right)=\chi\left(f\left(u_{f}\right), f\left(v_{f}\right)\right)=\chi\left(f^{-1}\left(u_{f}\right), f^{-1}\left(v_{f}\right)\right)=2$, yet $\mathcal{F}$ is not normal in $\mathbb{C}_{\infty}$.

Remark 2 It is known that if a sequence of Möbius maps converges at three distinct points to three distinct values, then it converges uniformly on $\mathbb{C}_{\infty}$ to a Möbius map [18]. We sketch a proof of this fact that is reminiscent of standard proofs of Vitali's theorem using a normal families argument. Let $f_{1}, f_{2}, \ldots$ be a sequence of Möbius maps. Suppose that $z_{1}, z_{2}, z_{3}$ and $w_{1}, w_{2}, w_{3}$ are two triples of distinct points in $\mathbb{C}_{\infty}$ and $f_{n}\left(z_{j}\right) \rightarrow w_{j}$, for $j=1,2,3$. Let $f$ be the unique Möbius map with $f\left(z_{j}\right)=w_{j}$, $j=1,2,3$. Theorem 1 implies that $\mathcal{F}=\left\{f_{n}: n=1,2, \ldots\right\}$ is a normal family. A sequence of functions from a normal family converges locally uniformly to a fixed limit function if and only if each subsequence has this same function as a local uniform limit. If a subsequence of $f_{1}, f_{2}, \ldots$ converges uniformly on $\mathbb{C}_{\infty}$, then the limit function is a Möbius map that takes $z_{1}$ to $w_{1}, z_{2}$ to $w_{2}$ and $z_{3}$ to $w_{3}$. Hence, the limit must be $f$ because a Möbius map is uniquely determined by its action on three points. A quantitative version of this result was established in [5].

\section{Locally Omitted Values and Normality}

Montel's three omitted values theorem tells us that a family of meromorphic functions on a region $\Omega$ is normal in $\Omega$ if it omits three distinct values. Because Möbius maps have no omitted values in $\mathbb{C}_{\infty}$ itself, there is no analog of Montel's three omitted values theorem for Möbius maps on $\mathbb{C}_{\infty}$. We recall a related result on omitted values for Möbius maps, where normality is now on a proper subregion of $\mathbb{C}_{\infty}$, see [3, p. 42].

Theorem 7 Let $\Omega$ be a proper subregion of $\mathbb{C}_{\infty}$, and let $u$ and $v$ be distinct points in $\mathbb{C}_{\infty}$. Then the family of Möbius maps that omit the values $u$ and $v$ in $\Omega$ is uniformly Lipschitz on compact subsets of $\Omega$.

Theorem 7 shows that a sufficient condition for normality of a family of Möbius maps on $\Omega$ is that the family omits two distinct values, and this result has a Carathéodory-type extension to variable omitted values provided they are uniformly separated. 
Theorem 8 Suppose that $\Omega$ is a proper subregion of $\mathbb{C}_{\infty}, \mathcal{F}$ is a family of Möbius maps, and for each $f \in \mathcal{F}$ there exist distinct points $u_{f}, v_{f} \in \mathbb{C}_{\infty}$ such that $f$ omits in $\Omega$ both $u_{f}$ and $v_{f}$. If the pairs $\left\{\left(u_{f}, v_{f}\right): f \in \mathcal{F}\right\}$ are uniformly separated, then $\mathcal{F}$ is uniformly Lipschitz on compact subsets of $\Omega$.

Proof Suppose that $\chi\left(u_{f}, v_{f}\right) \geq \varepsilon$ for all $f \in \mathcal{F}$, where $\varepsilon>0$. Then in $\Omega$ each $f \in \mathcal{F}$ satisfies the Lipschitz-type condition

$$
\chi(f(z), f(\zeta)) \leq \frac{8 \chi(z, \zeta)}{\varepsilon \sqrt{\chi(z, \partial \Omega)} \sqrt{\chi(\zeta, \partial \Omega)}}
$$

for all $z, \zeta \in \Omega$; see [3, p. 42]. This inequality implies that $\mathcal{F}$ is uniformly Lipschitz on each compact subset $K$ of $\Omega$ with explicit Lipschitz constant $8 /(\varepsilon \chi(K, \partial \Omega))$.

We are now in a position to prove Theorem 2.

Proof of Theorem 2 Suppose that (a) holds. Choose $z_{0} \in \Omega$ and let $K$ be a compact chordal disc centred at $z_{0}$ and contained in $\Omega$. Marty's theorem and (2.3) imply that there is a positive constant $M$ such that for all $z$ and $w$ in $K$ and all $f$ in $\mathcal{F}$, $\chi(f(z), f(w)) \leq M \chi(z, w)$. Let $\varepsilon$ be the minimum of $\chi(u, v), \chi(v, w)$ and $\chi(w, u)$. Consider an open chordal disc $D$ centred at $z_{0}$ and contained in $K$ with radius less than $\varepsilon /(2 M)$. Then for all $f \in \mathcal{F}$ and any $z_{1}, z_{2} \in D$,

$$
\chi\left(f\left(z_{1}\right), f\left(z_{2}\right)\right) \leq M \chi\left(z_{1}, z_{2}\right)<\varepsilon
$$

It follows that at most one of the values $u, v$ and $w$ is contained in $f(D)$. Thus (b) holds. Next, (c) follows trivially from (b). Finally, suppose that (c) holds. Then, by Theorem 8, each $z$ in $\mathbb{C}_{\infty}$ has an open neighbourhood on which $\mathcal{F}$ is normal. Because normality is a local property, $\mathcal{F}$ is normal in $\Omega$, which is statement (a).

By using Theorem 8 , it is possible to obtain a version of Theorem 2 involving variable triples of points.

\section{The Chordal Derivative and Normality}

The norm of a Möbius map can be expressed in terms of the chordal derivative at any pair of antipodal points. Recall that for any $z \in \mathbb{C}_{\infty}, z$ and $-1 / \bar{z}$ are a pair of antipodal points in $\mathbb{C}_{\infty}$.

Theorem 9 For any Möbius map $f$ and any $z \in \mathbb{C}_{\infty}$,

$$
\|f\|^{2}=\frac{1}{f^{\chi}(z)}+\frac{1}{f^{\chi}(-1 / \bar{z})} .
$$

Proof This is an elementary computation; add the identity (2.2) for $z$ to the identity with $z$ replaced with $-1 / \bar{z}$. 
Inequality (3.3) shows that if $\mathcal{F}$ is a normal family, then the family of chordal derivatives $\left\{f^{\chi}: f \in \mathcal{F}\right\}$ is uniformly bounded on $\mathbb{C}_{\infty}$. Using Theorem 9 we can obtain a stronger necessary and sufficient condition for normality.

Corollary 4 Let $\mathcal{F}$ be a family of Möbius maps. Then $\mathcal{F}$ is a normal family in $\mathbb{C}_{\infty}$ if and only if $\sup \left\{f^{\chi}(z): f \in \mathcal{F}, z \in f^{-1}(\{0, \infty\})\right\}<+\infty$.

Proof First, if $\mathcal{F}$ is a normal family, then $\left\{f^{\chi}: f \in \mathcal{F}\right\}$ is uniformly bounded on $\mathbb{C}_{\infty}$, so $\sup \left\{f^{\chi}(z): f \in \mathcal{F}, z \in f^{-1}(\{0, \infty\})\right\}<+\infty$. Conversely, suppose that there exists a positive constant $M$ with $f^{\chi}(z) \leq M$ for $z \in f^{-1}(\{0, \infty\})$ and all $f \in \mathcal{F}$. For $f \in \mathcal{F}$, consider $g=f^{-1}$. If $z_{0}=f^{-1}(0)$, then

$$
1=g^{\chi}\left(f\left(z_{0}\right)\right) f^{\chi}\left(z_{0}\right) \leq M g^{\chi}(0)
$$

so $g^{\chi}(0) \geq 1 / M$. Similarly, $g^{\chi}(\infty) \geq 1 / M$. Then Theorem 9 implies that $\|f\|^{2}=$ $\|g\|^{2} \leq 2 \bar{M}$. This holds for all $f \in \mathcal{F}$, so $\mathcal{F}$ is normal in $\mathbb{C}_{\infty}$.

Since we can pass from one uniformly separated set of pairs, or triples, to another without creating or destroying normality, the choice of 0 and $\infty$ here is not essential and these points can be replaced by any set of uniformly separated pairs, as is the case in Theorem 3.

Proof of Theorem 3 To show that (a) implies (b), suppose that $\mathcal{F}$ is normal in $\mathbb{C}_{\infty}$. Then $\left\{f^{\chi}: f \in \mathcal{F}\right\}$ is uniformly bounded on $\mathbb{C}_{\infty}$, say by $M$, and $f^{\chi}(z) \leq M$ for all $f \in \mathcal{F}$ and $z \in f^{-1}\left(\left\{u_{f}, v_{f}\right\}\right)$. Thus, (a) implies (b).

That (b) implies (c) is trivial.

Finally, we prove that (c) implies (a). By Corollary 3, we can construct a normal family of Möbius maps $\mathcal{H}=\left\{h_{f}: f \in \mathcal{F}\right\}$ on $\mathbb{C}_{\infty}$ such that, for all $f$, we have $h_{f}\left(u_{f}\right)=0$ and $h_{f}\left(v_{f}\right)=\infty$. Because $\mathcal{H}$ is normal, there is a finite constant $L$ such that $h^{\chi}(w) \leq L$ for all $w \in \mathbb{C}_{\infty}$ and all $h \in \mathcal{H}$. Let $z_{0}=f^{-1}\left(u_{f}\right)$. Then $h_{f} f\left(z_{0}\right)=0$, so we obtain the inequality

$$
\left(h_{f} f\right)^{\chi}\left(z_{0}\right)=h_{f}^{\chi}\left(u_{f}\right) f^{\chi}\left(z_{0}\right) \leq L M,
$$

where

$$
M=\sup \left\{f^{\chi}(z): f \in \mathcal{F}, z \in f^{-1}\left(\left\{u_{f}, v_{f}\right\}\right)\right\} .
$$

This inequality holds for all functions in $\mathcal{K}=\left\{h_{f} f: f \in \mathcal{F}\right\}$. Likewise, the same bound holds for all functions in $\mathcal{K}$ when $z_{0}=f^{-1}\left(v_{f}\right)$. Hence, Corollary 4 implies that $\mathcal{K}$ is a normal family. As the families $\mathcal{K}$ and $\mathcal{H}^{-1}$ are both normal and $f=h_{f}^{-1}\left(h_{f} f\right)$, Corollary 1 implies that $\mathcal{F}$ is normal in $\mathbb{C}_{\infty}$.

\section{A Geometric Perspective}

In this section we comment on the geometry that lies behind the results in this paper. First, let $\mathbb{H}^{3}=\left\{\left(x_{1}, x_{2}, x_{3}\right) \in \mathbb{R}^{3}: x_{3}>0\right\}$. Then $\mathbb{H}^{3}$, when equipped with the 
metric $d s=|d x| / x_{3}$, is a model of three-dimensional hyperbolic space, say with hyperbolic distance $\rho$. Further, the group of Möbius automorphisms of $\mathbb{H}^{3}$ is the group of conformal isometries of the associated hyperbolic geometry of $\mathbb{H}^{3}$. We then have the fundamental result (see [3, p. 61]) that

$$
\|f\|^{2}=2 \cosh \rho(\zeta, f(\zeta)), \quad \zeta=(0,0,1)
$$

Next, as we remarked earlier, both stereographic projection and its inverse $\Phi: \mathbb{C}_{\infty} \rightarrow \mathbb{S}^{2}$ are appropriate restrictions of the inversion $\mathcal{I}$ in the sphere $\left\{x \in \mathbb{R}^{3}:|x-\zeta|=\sqrt{2}\right\}$. Let $\mathcal{R}$ denote the Euclidean reflection in the plane $x_{3}=0$ (and recall that we have identified this plane with $\mathbb{C}$ ). Since $\mathcal{R}$ fixes each point of $\mathbb{C}_{\infty}$, we see that $\Phi$ is the restriction of the composite map $\mathcal{I} \circ \mathcal{R}$ to $\mathbb{C}_{\infty}$. This composite transformation maps $\mathbb{C}_{\infty}$ onto $\mathbb{S}^{2}$, and it maps the upper-half $\mathbb{H}^{3}$ of $\mathbb{R}^{3}$ onto the unit ball $\mathbb{B}^{3}$.

Now, corresponding to each Möbius map $f$ of $\mathbb{C}_{\infty}$ onto itself there is a map $f^{*}: \mathbb{S}^{2} \rightarrow \mathbb{S}^{2}$ which is given by $f^{*}=\Phi f \Phi^{-1}$; this simply transfers the action of $f$ on $\mathbb{C}_{\infty}$ to an action of $f^{*}$ on $\mathbb{S}^{2}$. Since the Möbius map $f$ acts on $\mathbb{H}^{3}$ as well as on $\mathbb{C}_{\infty}$, we see by thinking of $\Phi$ as $\mathcal{I} \circ \mathcal{R}$ that $f^{*}$ also acts on the unit ball $\mathbb{B}^{3}$, and this action is an isometry of $\mathbb{B}^{3}$ endowed with the hyperbolic metric $2|d x| /\left(1-|x|^{2}\right)$ and hyperbolic distance $\rho$ (for more details see, for example, $[1-3,19]$ ). Finally, since $\mathcal{I} \circ \mathcal{R}: \mathbb{H}^{3} \rightarrow \mathbb{B}^{3}$ is an isometry (of the two hyperbolic metrics), we obtain

$$
2 \cosh \rho\left(\mathbf{0}, f^{*}(\mathbf{0})\right)=2 \cosh \rho(\zeta, f(\zeta))=\|f\|^{2}, \quad \mathbf{0}=(0,0,0) .
$$

If we now return to (3.2), we find that the best Lipschitz constant for the action of $f$ on $\mathbb{C}_{\infty}$ is

$$
E(f)=\exp \rho\left(\mathbf{0}, f^{*}(\mathbf{0})\right)
$$

This description gives a visual explanation of Theorem 4; see Fig. 1, which indicates why a uniform bound on $\rho\left(\mathbf{0}, f^{*}(\mathbf{0})\right)$ imposes a uniform bound on the distortion of the action of $f^{*}$ on $\mathbb{S}^{2}$, and hence a bound on the distortion of $f$ in terms of $\chi$. Briefly, we consider the family of all hyperbolic geodesics in $\mathbb{B}^{3}$ that pass through $\mathbf{0}$. Their images under $f^{*}$ are the family of geodesics that pass through $f^{*}(\mathbf{0})$, and it is intuitively clear from Fig. 1 that if $\mathbf{0}$ is only moved a bounded distance, then the distortion on $\mathbb{S}^{2}$ is uniformly controlled.

However, Theorem 4 implies much more than this for, in conjunction with (7.1) and the Arzelà-Ascoli theorem, it shows that $\sup \{\|f\|: f \in \mathcal{F}\}<+\infty$ is not only a necessary and sufficient condition for a family $\mathcal{F}$ of Möbius maps to be normal on the boundary $\mathbb{S}^{2}$ of $\mathbb{B}^{3}$ (and hence on $\mathbb{C}_{\infty}$ ), it is also a necessary and sufficient condition for $\mathcal{F}$ to be normal in $\mathbb{B}^{3}$ itself. Finally, while Carathéodory (and others) use the inequalities $|a| \leq M,|b| \leq M,|c| \leq M$ and $|d| \leq M$ instead of a logically equivalent condition $\|f\| \leq M_{1}$, this conceals (rather than reveals) the underlying geometry because whereas $\|f\|$ has a clear geometric interpretation, the individual coefficients have no such interpretation. 

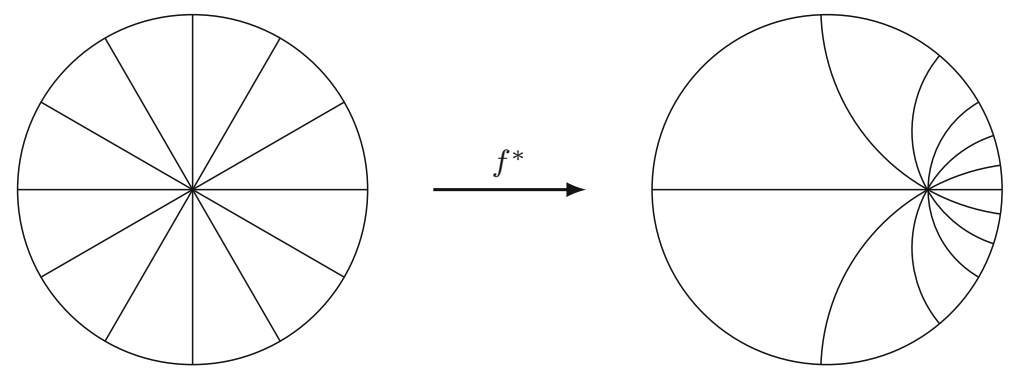

Fig. 1 The distortion on $\mathbb{S}^{2}$ is controlled by the hyperbolic distance between $\mathbf{0}$ and $f^{*}(\mathbf{0})$

\section{The Average of the Reciprocal of the Chordal Derivative}

From Theorem 9 we see that, for any Möbius map $f$, the average of the reciprocals of the chordal derivatives $f^{\chi}(0)$ and $f^{\chi}(\infty)$ is $\frac{1}{2}\|f\|^{2}$. The significance of 0 and $\infty$ here is that $\Phi(0)$ and $\Phi(\infty)$ are two points on $\mathbb{S}^{2}$ whose centre of gravity lies at the origin $\mathbf{0}$ in $\mathbb{R}^{3}$. Next, it is shown in [5] and in the proof of Theorem 5 that if $\omega_{1}, \omega_{2}$ and $\omega_{3}$ are the three cube roots of unity then, for a Möbius map $f$,

$$
\frac{1}{3}\left(\frac{1}{f \chi\left(\omega_{1}\right)}+\frac{1}{f \chi\left(\omega_{2}\right)}+\frac{1}{f \chi\left(\omega_{3}\right)}\right)=\frac{1}{2}\|f\|^{2}
$$

and the three points $\Phi\left(\omega_{1}\right), \Phi\left(\omega_{2}\right)$ and $\Phi\left(\omega_{3}\right)$ on $\mathbb{S}^{2}$ have centre of gravity $\mathbf{0}$. We shall now absorb these two results into a more general result which will show that, for certain probability measures $\mu$ on $\mathbb{C}_{\infty}$ the expected value of the reciprocal of the chordal derivative $f^{\chi}$ of $f$ with respect to $\mu$ is $\frac{1}{2}\|f\|^{2}$. We shall prove this for the simplest case of a probability measure $\mu$ that is uniformly distributed over a finite set of points $z_{1}, \ldots, z_{n}$; the general result follows in a similar way.

We start with a discrete uniform probability measure $\mu$ on $\mathbb{C}_{\infty}$, with a mass $1 / n$ at each of the points $z_{1} \ldots, z_{n}$ in $\mathbb{C}_{\infty}$, and then transfer this to the measure $\mu^{*}$ on $\mathbb{S}^{2}$ which has a mass of $1 / n$ at each of the points $\Phi\left(z_{1}\right), \ldots, \Phi\left(z_{n}\right)$. We need to assume that the measure $\mu^{*}$ has its Euclidean centre of gravity at $\mathbf{0}$, and with this we can prove the following result.

Lemma 1 Let $f$ be a Möbius map, and let $z_{1}, \ldots, z_{n}$ be points in $\mathbb{C}_{\infty}$ such that $\Phi\left(z_{1}\right)+\cdots+\Phi\left(z_{n}\right)=\mathbf{0}$. Then

$$
\frac{1}{n}\left(\frac{1}{f^{\chi}\left(z_{1}\right)}+\frac{1}{f^{\chi}\left(z_{2}\right)}+\cdots+\frac{1}{f^{\chi}\left(z_{n}\right)}\right)=\frac{1}{2}\|f\|^{2}=\cosh \rho\left(\mathbf{0}, f^{*}(\mathbf{0})\right) .
$$

Proof First, the identity $\cosh \rho\left(\mathbf{0}, f^{*}(\mathbf{0})\right)=\frac{1}{2}\|f\|^{2}$ was discussed in Sect. 7.

Next, the points $z_{j}$ are in $\mathbb{C}_{\infty}$, and $\sum_{j} \Phi\left(z_{j}\right)=\mathbf{0}$. Thus, from (2.1), we see that

$$
\frac{z_{1}}{\left|z_{1}\right|^{2}+1}+\frac{z_{2}}{\left|z_{2}\right|^{2}+1}+\cdots+\frac{z_{n}}{\left|z_{n}\right|^{2}+1}=0
$$


and

$$
\frac{\left|z_{1}\right|^{2}-1}{\left|z_{1}\right|^{2}+1}+\frac{\left|z_{2}\right|^{2}-1}{\left|z_{2}\right|^{2}+1}+\cdots+\frac{\left|z_{n}\right|^{2}-1}{\left|z_{n}\right|^{2}+1}=0 .
$$

Since (trivially)

$$
\frac{\left|z_{1}\right|^{2}+1}{\left|z_{1}\right|^{2}+1}+\frac{\left|z_{2}\right|^{2}+1}{\left|z_{2}\right|^{2}+1}+\cdots+\frac{\left|z_{n}\right|^{2}+1}{\left|z_{n}\right|^{2}+1}=n,
$$

we see that

$$
\frac{\left|z_{1}\right|^{2}}{\left|z_{1}\right|^{2}+1}+\cdots+\frac{\left|z_{n}\right|^{2}}{\left|z_{n}\right|^{2}+1}=\frac{1}{\left|z_{1}\right|^{2}+1}+\cdots+\frac{1}{\left|z_{n}\right|^{2}+1}=\frac{1}{2} n .
$$

Finally, with $f(z)=(a z+b) /(c z+d)$, where $a d-b c=1$, we have

$$
\begin{aligned}
\frac{1}{n} \sum_{j=1}^{n} \frac{1}{f^{\chi}\left(z_{j}\right)} & =\frac{1}{n} \sum_{j=1}^{n} \frac{\left|a z_{j}+b\right|^{2}+\left|c z_{j}+d\right|^{2}}{\left|z_{j}\right|^{2}+1} \\
& =\frac{1}{n} \sum_{j=1}^{n} \frac{\left(|a|^{2}+|c|^{2}\right)\left|z_{j}\right|^{2}+\left(|b|^{2}+|d|^{2}\right)+(a \bar{b}+c \bar{d}) z_{j}+(\bar{a} b+\bar{c} d) \overline{z_{j}}}{\left|z_{j}\right|^{2}+1} \\
& =\frac{1}{2}\left(|a|^{2}+|b|^{2}+|c|^{2}+|d|^{2}\right) \\
& =\frac{1}{2}\|f\|^{2},
\end{aligned}
$$

as required.

We can now consider the general situation. We shall say that a Borel probability measure $\mu$ on $\mathbb{C}_{\infty}$ is admissible if the corresponding push-forward measure $\mu^{*}$ on $\mathbb{S}^{2}$, defined by $\mu^{*}(E)=\mu\left(\Phi^{-1}(E)\right)$, satisfies

$$
\int_{\mathbb{S}^{2}} \mathbf{x} d \mu^{*}(\mathbf{x})=\mathbf{0}
$$

equivalently, the measure $\mu^{*}$ on $\mathbb{S}^{2}$ has its Euclidean centre of gravity at the origin $\mathbf{0}$ in $\mathbb{R}^{3}$. We then have the following result.

Theorem 10 Let $f$ be a Möbius map, and let $\mu$ be an admissible probability measure on $\mathbb{C}_{\infty}$. Then the expected value of $1 / f^{\chi}$ with respect to $\mu$ over $\mathbb{C}_{\infty}$ is $\frac{1}{2}\|f\|^{2}$; that is,

$$
\int_{\mathbb{C}_{\infty}} \frac{1}{f^{\chi}(z)} d \mu(z)=\frac{1}{2}\|f\|^{2}=\cosh \rho\left(\mathbf{0}, f^{*}(\mathbf{0})\right) .
$$

We leave the reader to provide the proof of Theorem 10, which mirrors the proof of Lemma 1 after replacing the sums by integrals. 
Open Access This article is licensed under a Creative Commons Attribution 4.0 International License, which permits use, sharing, adaptation, distribution and reproduction in any medium or format, as long as you give appropriate credit to the original author(s) and the source, provide a link to the Creative Commons licence, and indicate if changes were made. The images or other third party material in this article are included in the article's Creative Commons licence, unless indicated otherwise in a credit line to the material. If material is not included in the article's Creative Commons licence and your intended use is not permitted by statutory regulation or exceeds the permitted use, you will need to obtain permission directly from the copyright holder. To view a copy of this licence, visit http://creativecommons.org/licenses/by/4.0/.

\section{References}

1. Ahlfors, L.V.: Hyperbolic motions. Nagoya Math. J. 29, 163-166 (1967)

2. Ahlfors, L.V.: Möbius transformations in several dimensions, Ordway Professorship Lectures in Mathematics. University of minnesota, Minneapolis (1981)

3. Beardon, A.F.: The geometry of discrete groups, Graduate Texts in Mathematics, 91. Springer, New York (1983)

4. Beardon, A.F., Lorentzen, L.: Continued fractions and restrained sequences of Möbius maps. Rocky Mt. J. Math. 34(2), 441-466 (2004)

5. Beardon, A.F., Short, I.: Norms of Möbius maps. Bull. Lond. Math. Soc. 42(3), 499-505 (2010)

6. Beardon, A.F., Minda, D.: Normal families: a geometric perspective. Comput. Methods Funct. Theory Gehr. Mem. 14, 331-355 (2014)

7. Beardon, A.F., Minda, D.: An extension of Montel's three omitted values theorem (submitted)

8. Carathéodory, C.: Theory of functions of a complex variable, vol. II. Chelsea, New York (1958)

9. Chuang, C.T.: Normal families of meromorphic functions. World Scientific Publishing, New Jersey (1993)

10. Hinkkanen, A.: Normal families and Ahlfors' five islands theorem. New Zealand J. Math. 22, 39-41 (1993)

11. Lappan, P.: A criterion for a meromorphic function to be normal. Comment. Math. Helv. 49, 492-495 (1974)

12. Lappan, P.: A uniform approach to normal families. Rev. Roumaine Math. Pures Appl. 39(7), 691-702 (1994)

13. Marty, F.: Recherches sur la répartition des valeurs d'une fonction méromorphe. Ann. Fac. Sci. Toulous. 23, 183-261 (1931)

14. Montel, P.: Lecons sur les familles normales de fonctions analytiques et leurs applications. GauthierVillars, Paris (1927)

15. Montel, P.: Lecons sur les familles normales de fonctions analytiques et leurs applications, 2nd edn. Chelsea Pub. Co., New York (1974)

16. Montel, P.: Sur un critère principal de normalité. J. Anal. Math. 3, 209-224 (1954)

17. Pedoe, D.: On some geometrical inequalities. Math. Gaz. 26, 202-208 (1942)

18. Piranian, G., Thron, W.J.: Convergence properties of sequences of linear fractional transformations. Mich. Math. J. 4, 129-135 (1957)

19. Ratcliffe, J.G.: Foundations of hyperbolic manifolds, graduate texts in mathematics, vol. 149. Springer, New York (1994)

20. Schiff, J.L.: Normal families. Springer-Verlag, New York (1993)

21. Weitzenböck, R.: Über eine Ungleichung in der Dreiecksgeometrie. Math. Z. 5, 137-146 (1919)

Publisher's Note Springer Nature remains neutral with regard to jurisdictional claims in published maps and institutional affiliations. 